\title{
Tarifpolitischer Halbjahresbericht: Eine Zwischenbilanz der Lohn- und Gehaltsrunde 2013
}

\author{
Die Tarifrunde 2013 ist bereits zur Jahresmitte in weiten Teilen abgeschlossen. Für viele \\ große und auch kleinere Branchen und Tarifbereiche liegen Abschlüsse vor, die eine fun- \\ dierte Zwischenbilanz des Tarifjahres erlauben. Die Ergebnisse zeigen, dass sich zwar \\ die Abschlussraten im Vergleich zum Vorjahr etwas verringert haben, die jahresbezogene \\ Tariferhöhung hat sich dennoch geringfügig erhöht. Der neutrale Verteilungsspielraum \\ wurde bislang ausgeschöpft. Die Tariflöhne und -gehälter werden in diesem Jahr preisbe- \\ reinigt voraussichtlich um etwa $1 \%$ steigen.
}

REINHARD BISPINCK, WSI-TARIFARCHIV

\section{1. Übersicht}

Die wirtschaftlichen Rahmenbedingungen der Tarifrunde waren durchwachsen: Ähnlich wie bereits im vergangenen Jahr hatte sich die konjunkturelle Entwicklung in Deutschland im Vorfeld deutlich verschlechtert. Im letzten Quartal 2012 war das Bruttoinlandsprodukt (BIP) um 0,6 \% zurückgegangen. Die Entwicklung auf dem Arbeitsmarkt war 2012 auf den ersten Blick stabil, der Rückgang der Arbeitslosigkeit beruhte jedoch allein auf der guten Entwicklung des vergangenen Jahres, die Arbeitslosigkeit stieg im Jahresverlauf und auch im ersten Halbjahr 2013 an (BA 2012, S. 47). Für das laufende Jahr sahen die Konjunkturprognosen nur ein schwaches Wachstum von unter einem Prozent vorher. Die zum Teil massive Rezession in Europa, die von den südeuropäischen Krisenländern auf weitere Länder wie Frankreich, Niederlande und Großbritannien übergriff, beeinflusste die hiesige Debatte nur am Rande. Während die Mainstream-Ökonomen einen lohnpolitischen Verteilungsspielraum von rund $2 \%$ sahen, plädierten keynesianische Ökonomen wie Peter Bofinger für einen Zuschlag von $2 \%$ zur Euro-Rettung und sprachen sich für gesamtwirtschaftliche Lohnsteigerungen von rund $5 \%$ aus. ${ }^{1}$ Diese stärkere Lohnsteigerung sollte über die wachsende Binnennachfrage eine Stabilisierungsfunktion für die europäische Wirtschaftsentwicklung insgesamt wahrnehmen. Eine stärkere Lohnentwicklung in Deutschland ist auch längerfristig zwingend erforderlich, um die großen Leistungsbilanzungleichgewichte im Euroraum schneller abzubauen (Stein et al. 2012). In der lohn- und verteilungspolitischen Diskussion spielten im Umfeld der diesjährigen Tarifrunde die wachsenden Probleme im Niedriglohnsektor und das Lohndumping in bestimmten Branchen ebenso eine Rolle wie die generelle Spreizung der Einkommens- und Vermögensverhältnisse (Brenke/Wagner 2013).

Die Lohn- und Gehaltsforderungen der Gewerkschaften bewegten sich in der diesjährigen Tarifrunde zwischen 5 und 6,6\% und damit auf etwas niedrigerem Niveau als im Vorjahr. Das wichtigste Signal kam zweifellos von der IG Metall. Mit ihrer Tarifforderung von 5,5 \% für zwölf Monate für die Metallindustrie blieb sie einen Prozentpunkt unter ihrer Vorjahresforderung. Daraus konnte man entnehmen, dass ein Abschluss in Vorjahreshöhe nicht um jeden Preis angestrebt wurde. Ebenfalls 5,5\% wurden gefordert bei Volkswagen und im Kfz-Gewerbe. Darunter blieben noch die Eisen- und Stahlindustrie, die Holz- und Kunststoffindustrie und die ostdeutsche Textilindustrie mit einer gewerkschaftlichen Forderung von jeweils 5,0\%. Die IG Metall positionierte sich also in verschiedenen Bran-

1 Spiegel Online: „Peter Bofinger: Wirtschaftsweiser fordert Lohnplus von fünf Prozent,' http://www.spiegel.de/ wirtschaft/unternehmen/peter-bofinger-wirtschaftsweiserfordert-lohnplus-von-fuenf-prozent-a-875948.html. 
ÜBERSICHT 1

Ausgewählte Tarifforderungen und -abschlüsse in der Tarifrunde 2013
Abschluss Tarifbereich
Forderung
Lohn, Gehalt, Entgelt

2013

2014

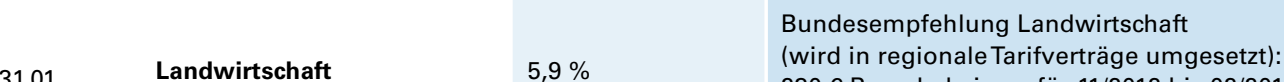

$230 €$ Pauschale insg. für $11 / 2012$ bis $02 / 2013$

$2,8 \%$ ab $07 / 2014$ bis $06 / 2015$

$3,7 \%$ ab 03/2013

\begin{tabular}{lll}
06.02 & $\begin{array}{l}\text { Tarifgemeinschaft } \\
\text { Energie (u. a. E.ON) }\end{array}$ & $6,5 \%$ \\
\hline 25.02. & $\begin{array}{l}\text { Holz- und Kunststoff } \\
\text { Westfalen-Lippe }\end{array}$ & $5,0 \%$ \\
\hline 25.02. & Tarifgruppe RWE & $6,0 \%$ \\
\hline 01.03. & Steinkohlenbergbau & Reallohnzuwachs \\
\hline
\end{tabular}

2,8 \% ab 01/2013 bis 01/2014

$300 €$ zusätzliche Einmalzahlung

2 Nullmonate

$3,0 \%$ ab $03 / 2013$ bis $04 / 2014$

$250 €$ Pauschale für 01/2013

$2,75 \%$ ab 02/2013

$1,75 \%$ ab $02 / 2014$ bis $12 / 2014$

$630 €$ Pauschale insg. für 01 bis $03 / 2013$

$3,5 \%$ ab $04 / 2013$ bis $12 / 2014$

Stahlindustrie NRW

Stahlindustrie NRW,
Niedersachsen und Bremen $\quad 5,0 \%$

$3,0 \%$ ab $03 / 2013$ bis $05 / 2014$

09.03. Öffentlicher Dienst Länder

$6,5 \%$ mit sozialer Komponente

$2,65 \%$ ab $01 / 2013$

$2,95 \%$ ab $01 / 2014$ bis $12 / 2014$

$\begin{array}{ll}\text { Energiewirtschaft } \\ \text { 11.03. } & \text { Ost (AVEU) }\end{array}$

$6,5 \%$

$550 €$ Pauschale insg. für 03 bis $04 / 2013$

$1,8 \%$ ab 05/2014 bis 04/2015

2,8 \% ab 05/2013

$275 €$ zusätzliche Einmalzahlung

25.03. Deutsche Bahn AG

$6,5 \%$

$500 €$ Pauschale insg. für 01 bis $04 / 2013$

3,0 \% ab 05/2013

$3,0 \%$ ab $04 / 2014$ bis $07 / 2014$

05.04

Bauhauptgewerbe

$6,6 \%$

1 Nullmonat

3,2 \% West, Berlin-Ost

4,0\% Ost

jew. ab 05/2013 bis 04/2014

unterste Gruppe:

$5,8 \%$ ab 05/2013

$4,4 \%$ ab $01 / 2014$ bis $12 / 2014$

07.04

Bewachungsgewerbe NRW rd. $30 \%$ *

Aviation:

$10,0 \%$ ab 05/2013

$8,1 \%$ ab $01 / 2014$ bis $12 / 2014$

$45 €$ Pauschale für $03 / 2013$

08.04 Hotels und Gaststätten

$120 €$

$4,0 \%$ ab $04 / 2013$ bis $07 / 2014$

Priv. Transport u. Verkehr

08.04. NRW

$6,5 \%$, mind. $125 €$

3 Nullmonate

2,8 \% ab 06/2013 bis 05/2014

10.04

Kautschukindustrie

2,1\% ab 05/2013

$1,1 \%$ ab 01/2014 bis $06 / 2014$

$2,7 \%$ ab $02 / 2013$

$2,4 \%$ ab $02 / 2014$ bis $12 / 2014$

$60 €$ Pauschale für $04 / 2013$

19.04 Textilindustrie Ost

$5,0 \%$

$3,0 \%$ ab 05/2013

$2,6 \%$ ab $07 / 2014$ bis $03 / 2015$

26.04. Deutsche Post AG

$6,0 \%$

4 Nullmonate

mind. $140 € \quad 3,1 \%$ ab 08/2013

$2,6 \%$ ab $10 / 2014$ bis $05 / 2015$

(Mindesterhöhung von $2.200 €$ je

AN bezogen auf die Laufzeit)

\begin{tabular}{|c|c|c|c|}
\hline 14.05. & Metallindustrie & $5,5 \%$ & $\begin{array}{l}2 \text { Nullmonate } \\
3,4 \% \text { ab } 07 / 2013\end{array}$ \\
\hline 22.05. & $\begin{array}{l}\text { Kfz-Gewerbe } \\
\text { Bayern }\end{array}$ & $5,5 \%$ & $\begin{array}{l}2 \text { Nullmonate } \\
2,8 \% \text { ab } 07 / 2013\end{array}$ \\
\hline 28.05. & Volkswagen AG & $5,5 \%$ & $\begin{array}{l}2 \text { Nullmonate } \\
3,4 \% \text { ab } 09 / 2013 \\
275 € \text { Einmalzahlung bzw. } 300 € \text { Altersvorsorgebe- } \\
\text { trag }\end{array}$ \\
\hline 07.06 & Versicherungsgewerbe & $\begin{array}{l}6,5 \% \\
\text { mind. } 160 €\end{array}$ & $\begin{array}{l}4 \text { Nullmonate } \\
3,2 \% \text { ab } 08 / 2013\end{array}$ \\
\hline
\end{tabular}

$2,2 \%$ ab $05 / 2014$ bis $12 / 2014$

$2,8 \%$ ab 08/2014 bis $04 / 2015$

$2,2 \%$ ab 07/2014 bis 02/2015

$2,2 \%$ ab $10 / 2014$ bis $03 / 2015$ 
ÜBERSICHT 1

\section{Fortsetzung}

Abschluss Tarifbereich Forderung Lohn, Gehalt, Entgelt

2013

2014

\begin{tabular}{|c|c|c|c|c|}
\hline 14.06. & $\begin{array}{l}\text { Groß- und Außenhandel } \\
\text { Baden-Württemberg }\end{array}$ & $\begin{array}{l}6,5 \% \\
\text { mind. } 140 €\end{array}$ & $\begin{array}{l}2 \text { Nullmonate } \\
3,0 \% \text { ab 06/2013 }\end{array}$ & $\begin{array}{l}2,1 \% \text { ab } 04 / 2014 \text { bis } 03 / 2015 \\
90 € \text { Einmalzahlung }\end{array}$ \\
\hline 20.06. & Gebäudereinigerhandwerk & $\begin{array}{l}\text { uG: } 2 \text { Stufen } \\
\text { W: } 70 / 65 \text { ct. } \\
\text { O: } 98 / 98 \text { ct. }\end{array}$ & 2 Nullmonate & $\begin{array}{l}3,4 / 5,3 \% \text { ab 01/2014 (West/Ost) } \\
2,6 / 3,1 \% \text { ab 01/2015 (West/Ost) }\end{array}$ \\
\hline
\end{tabular}

* Unterste Lohngruppe: $+2,50 €$ (bislang: 8,15 $€ /$ Std.), Aviation: von $12,15 €$ auf $16,00 € /$ Std.

Quelle:WSI-Tarifarchiv; Stand: 20. Juni 2013

chen und Tarifbereichen am unteren Rand des Forderungsspektrums. Das führte allerdings, wie die Ergebnisse zeigen, nicht zwingend zu niedrig(er)en Abschlüssen. Die NGG forderte wie bereits 2012 für ihre Branchen zwischen 5 und $6 \%$ sowie tarifliche Mindestentgelte von 8,50€. Um die $6 \%$ forderten die Gewerkschaften in der Kautschukindustrie (5,8 \%), in der Landwirtschaft (5,9\%), im Energiebereich (RWE) und bei der Deutschen Post AG (jeweils 6,0 \%). In vielen weiteren Branchen belief sich die Tarifforderung auf 6,5 \% (Übersicht 1). Die IG BAU lag mit ihrer Forderung von 6,6\% für das Bauhauptgewerbe an der Spitze der größeren Tarifbranchen. In vielen Branchen wurden auch soziale Komponenten in Form von Mindesterhöhungen gefordert. In einzelnen Fällen wurden auch Tarifforderungen in Form von festen Eurobeträgen aufgestellt.

Zwei Branchen fielen mit ihrem Forderungsvolumen aus dem Rahmen üblicher Lohnrunden. In beiden Fällen handelt es sich um ausgesprochene Niedriglohnbranchen: Im Gebäudereinigerhandwerk forderte die IG BAU eine Erhöhung der untersten Stundenlöhne in zwei Stufen um 70/65 ct (West) bzw. 98/98 ct (Ost), was einer Erhöhung um knapp $15 \%$ (West) bzw. knapp 26 \% (Ost) im Zeitraum von zwei Jahren entspricht. Im Bewachungsgewerbe NordrheinWestfalen ging ver.di noch weiter: Die Gewerkschaft forderte für die unterste Lohngruppe (8,15 $€ /$ Std.) eine Erhöhung um 2,50 €, was einer Steigerung um 30,7 \% entspricht. Erklärtes Ziel war es, die Branche mit ihren Einkommen aus dem Niedriglohnsektor herauszuführen (siehe Abschnitt 3.3).

Neben den reinen Entgeltforderungen spielten in einigen Tarifbereichen auch qualitative Tarifforderungen eine Rolle: Sie bezogen sich u. a. auf die Übernahme der Ausgebildeten, die Weiterentwicklung von Demografie-Tarifverträgen, die Fortschreibung von Altersteilzeitregelungen und die betriebliche Altersversorgung.

Der Kündigungsterminkalender gab folgenden zeitlichen Ablauf der Tarifrunde vor:

- Ende Dezember 2012 liefen die Tarifverträge für den öffentlichen Dienst (Länder), für die Deutsche Bahn AG, die
Wohnungswirtschaft, die Holz und Kunststoff verarbeitende Industrie und einige Energiekonzerne aus.

- Im Februar 2013 endete die Laufzeit der Verträge in der Eisen- und Stahlindustrie und in weiteren Bereichen der Energiewirtschaft.

- Ende März folgten das Bauhauptgewerbe, das Versicherungsgewerbe und einige Bereiche des Einzelhandels und des Groß- und Außenhandels.

- Ende April standen die Verträge in der Metall- und Elektroindustrie, in der Kautschukindustrie, in weiteren Bereichen des Einzel- und Großhandels sowie des Kfz-Gewerbes zur Verhandlung an.

- Ende Mai folgten die Papierindustrie, das Textilreinigungsgewerbe und weitere Bereiche des Kfz-Gewerbes und des ostdeutschen Einzelhandels.

- Im Juni standen u. a. die Tarifverhandlungen für das Volkswagenwerk auf dem Tarifkalender.

Wegen der lang laufenden Abschlüsse aus den Vorjahren gibt es in einigen Branchen in diesem Jahr keine Lohnrunde. Das gilt beispielsweise für den öffentlichen Dienst (Bund und Gemeinden), das Bankgewerbe, die chemische Industrie, die Deutsche Telekom AG und zahlreiche kleinere Branchen (siehe WSI-Kündigungsterminkalender: www. boeckler.de/38687_40912.htm). Ein Blick auf die Tarifabschlüsse zeigt für das erste Halbjahr folgendes Bild:

\section{Februar:}

- Die ersten Abschlüsse erfolgten in verschiedenen Bereichen der Energiewirtschaft. Am 6.2. vereinbarten ver.di und IG BCE mit der Tarifgemeinschaft Energie (u. a. E.ON) eine Tarifsteigerung von 2,8 \% ab 1.1.2013 und eine zusätzliche Einmalzahlung von $300 €$ bei einer Gesamtlaufzeit von 13 Monaten.

- Für RWE wurden am 25.2. eine Pauschalzahlung von $250 €$, eine Tarifsteigerung von 2,75\% ab 1.2.2013 und eine Stufenanhebung von 1,75 \% ab 1.2.2014 mit einer Laufzeit von insgesamt 24 Monaten vereinbart. 
März:

- Am 6.3. erreichte die IG Metall in der westdeutschen Eisenund Stahlindustrie einen Abschluss mit einer Tarifsteigerung von 3,0 \% ab 1.3.2013 mit einer Laufzeit von 15 Monaten.

- Im öffentlichen Dienst (Länder) erfolgte der Abschluss nach kräftigen Warnstreiks am 9.3. in der dritten Verhandlungsrunde: Er sieht eine Tariferhöhung von 2,65 \% rückwirkend ab dem 1.1. vor, eine Stufenerhöhung von 2,95 \% ab dem 1.1.2014 bei einer Laufzeit von insgesamt 24 Monaten (siehe Abschnitt 3.1).

- Für die Deutsche Bahn AG vereinbarte die Eisenbahn- und Verkehrsgewerkschaft (EVG) am 25.3. eine Pauschalzahlung von $500 €$ insgesamt für Januar bis April, eine Tarifanhebung von 3,0 \% ab 1.5.2013 und eine Stufenanhebung von $3,0 \%$ ab 1.4.2014 bei einer Gesamtlaufzeit von 19 Monaten.

April:

- Die IG BAU vereinbarte für das Bauhauptgewerbe am 5.4. in der dritten Verhandlungsrunde nach einem Nullmonat eine Tarifanhebung von 3,2\% (West) und 4,0\% (Ost) ab 1.5.2013 mit einer Laufzeit von 13 Monaten.

- Am 7.4. wurde der heftige und lang anhaltende Tarifkonflikt im Bewachungsgewerbe Nordrhein-Westfalen durch Schlichtung beigelegt. Ver.di konnte für die unterste Lohngruppe eine Anhebung der Tarifvergütung um 10,5 \% und für die Passagierkontrolle um 18,9 \% (!) jeweils in einem Zeitraum von zwei Jahren durchsetzen. Dies stellt zweifelsohne den höchsten Abschluss der diesjährigen Tarifrunde dar.

- Im privaten Transport- und Verkehrsgewerbe NRW umfasste der Abschluss vom 8.4. eine Tarifanhebung nach drei Nullmonaten von 2,8 \% ab 1.6.2013 bei einer Laufzeit von 15 Monaten.

- Ebenfalls am 8.4. erfolgte der Abschluss im Hotel- und Gaststättengewerbe Bayern mit einer Pauschale von $45 €$ für den März und einer Tarifsteigerung von 4,0 \% ab 1.4.2013 bei einer Laufzeit von 17 Monaten.

- In der Kautschukindustrie erreichte die IG BCE am 10.4. eine Tarifanhebung von 2,1 \% ab 1.5.2013 und eine Stufenanhebung von weiteren 1,1 \% ab 1.1.2014 mit einer Laufzeit von 26 Monaten.

- Für die Deutsche Post $A G$ vereinbarte ver.di nach mehrtägigen Warnstreiks am 26.4. nach vier Nullmonaten (April bis Juli) eine Tarifanhebung von 3,1\% ab 1.8.2013 sowie eine Stufenerhöhung von 2,6 \% ab 1.10.2014. Die Laufzeit beträgt 26 Monate. Die Tarifbeschäftigten sollen mindestens eine Tariferhöhung von $2.200 €$ bezogen auf die Laufzeit erhalten, ggf. erfolgt eine ergänzende Einmalzahlung.

Mai:

- In der Metall- und Elektroindustrie vereinbarte die IG Metall nach umfangreichen Warnstreiks im gesamten Bundesgebiet am 14.5. einen Pilotabschluss in Bayern, der nach zwei Nullmonaten eine Tarifsteigerung von $3,4 \%$ ab
1.7.2013 und eine Stufenanhebung von weiteren 2,2 \% ab 1.5.2014 umfasst. Die gesamte Laufzeit beträgt 20 Monate. Der Abschluss für die Volkswagen AG vom 28.5. hat ein vergleichbares Volumen und enthält zusätzlich einen Rentenbaustein von $300 €$.

- Im Kfz-Gewerbe erfolgte der erste Abschluss am 22.5. ebenfalls für Bayern. Auch hier gab es zunächst zwei Nullmonate, anschließend ab 1.7. eine Tarifsteigerung von $2,8 \%$ und weitere 2,8 \% ab 1.8.2014 bei einer Laufzeit von 24 Monaten.

Juni:

- Für das Versicherungsgewerbe erreichte ver.di am 7.6. ein Tarifergebnis, das nach vier Nullmonaten eine Erhöhung der Tarifvergütungen um 3,2 \% ab 1.8.2013 und um weitere 2,2 \% ab 1.10.2014 vorsieht. Die Gesamtlaufzeit beträgt 24 Monate.

- Im Groß- und Außenhandel beinhaltet der erste regionale Abschluss für Baden-Württemberg vom 14.6. nach zwei Nullmonaten eine Tarifanhebung um 3,0 \% ab 1.6.2013 sowie eine weitere Anhebung um 2,1\% ab 1.4.2014 und eine zusätzliche Einmalzahlung.

- Am 20.6. schließlich erreichte die IG BAU nach monatelangen Verhandlungen in der fünften Runde für das Gebäudereinigerhandwerk folgenden Abschluss: eine Tarifanhebung nach zwei Nullmonaten um 3,4/5,3 \% ab 1.1.2014 und weitere 2,6/3,1 \% ab 1.1.2015 (jeweils West/Ost). Das Tarifniveau Ost wird dadurch von 84 auf $86 \%$ angehoben. Bis spätestens Januar 2019 soll die Tarifangleichung vollständig realisiert sein.

Nicht abgeschlossen war zum Redaktionsschluss die Tarifrunde im Einzelhandel. Hier geht es nicht nur um eine normale Lohnrunde, denn die Arbeitgeberverbände hatten im Vorfeld bundesweit alle Manteltarifverträge gekündigt (siehe Abschnitt 4).

\section{Ergebnisse im gesamtwirtschaftlichen Überblick}

Im 1. Halbjahr des Jahres 2013 schlossen die Gewerkschaften des Deutschen Gewerkschaftsbundes (DGB) Einkommenstarifverträge für rund 8,7 Mio. Beschäftigte ab, darunter rund 1,2 Mio. in den neuen Bundesländern. Das entspricht etwa $45 \%$ der von Tarifverträgen erfassten Arbeitnehmerinnen und Arbeitnehmer.

Die durchschnittliche Abschlussrate einschließlich aller Stufenanhebungen, die während der Laufzeit der Verträge wirksam werden, beträgt 5,3\% (West: 5,2 \%, Ost: 5,6 \%). Berücksichtigt werden bei der Abschlussrate nur die tabellenwirksamen Tarifanhebungen, während Pauschal- und zusätzliche Einmalzahlungen außen vor bleiben.

Berücksichtigt man ausschließlich die im Jahr 2013 in Kraft tretenden Tariferhöhungen, ergibt sich eine Abschluss- 
TABELLE 1

\section{Tarifsteigerung 2013'}

Angaben in absoluten Zahlen und in Prozent

\begin{tabular}{|c|c|c|c|c|c|c|}
\hline \multirow{2}{*}{ Wirtschaftsbereich } & \multicolumn{2}{|l|}{ West } & \multicolumn{2}{|l|}{ Ost } & \multicolumn{2}{|c|}{ Gesamt } \\
\hline & AN in 1000 & $\%$ & AN in 1000 & $\%$ & AN in 1000 & $\%$ \\
\hline Gartenbau, Land- und Forstwirtschaft & 83,0 & 2,7 & 61,8 & 3,1 & 144,8 & 2,9 \\
\hline Energie- und Wasserversorgung, Bergbau & 119,6 & 3,7 & 22,5 & 3,5 & 142,1 & 3,6 \\
\hline Grundstoff- und Produktionsgütergewerbe & 727,2 & 2,4 & 85,3 & 3,0 & 812,5 & 2,5 \\
\hline Investitionsgütergewerbe & $3.764,4$ & 3,0 & 378,0 & 3,1 & $4.142,4$ & 3,0 \\
\hline Verbrauchsgütergewerbe & 754,5 & 2,4 & 130,7 & 2,7 & 885,2 & 2,4 \\
\hline Nahrungs- und Genussmittelgewerbe & 274,9 & 2,8 & 22,2 & 2,8 & 297,1 & 2,8 \\
\hline Baugewerbe & 610,5 & 3,1 & 162,3 & 4,1 & 772,8 & 3,3 \\
\hline Handel & 554,7 & 2,5 & 67,3 & 2,5 & 622,0 & 2,5 \\
\hline Verkehr und Nachrichtenübermittlung & 737,6 & 2,4 & 91,5 & 2,6 & 829,1 & 2,4 \\
\hline Kreditinstitute, Versicherungsgewerbe & 386,8 & 2,3 & 30,7 & 2,3 & 417,5 & 2,3 \\
\hline Private Dienstleistungen, Org. o. Erwerbszweck & $1.260,6$ & 2,7 & 298,8 & 4,3 & $1.559,4$ & 3,0 \\
\hline Gebietskörperschaften, Sozialversicherung & $2.054,2$ & 2,6 & 516,9 & 2,6 & $2.571,1$ & 2,6 \\
\hline Gesamte Wirtschaft & $11.328,0$ & 2,7 & $1.868,0$ & 3,2 & $13.196,0$ & 2,8 \\
\hline
\end{tabular}

1 Jahresbezogene Erhöhung 2013 gegenüber 2012.

Quelle:WSI-Tarifarchiv; Stand: 20.06.2013.

rate von 3,2 \% (West: 3,2\%, Ost: 3,2\%). Damit liegt die Abschlussrate, die 2013 wirksam wird, um 0,7 Prozentpunkte niedriger als im Vorjahr (2012:3,9\%). Differenziert man diese Größe nach Wirtschaftsbereichen, dann ergibt sich in diesem Jahr folgende Streuung: Am unteren Ende liegt der Bereich Gebietskörperschaften, Sozialversicherung mit 2,7 \%, gefolgt von den Bereichen Energie- und Wasserversorgung, Bergbau sowie Verkehr und Nachrichtenübermittlung mit 2,9\%. Mit 3,0 \% schließen sich folgende Bereiche an: Grundstoff- und Produktionsgütergewerbe, Verbrauchsgütergewerbe, Nahrungs- und Genussmittelgewerbe und Handel. Der Bereich Kreditinstitute/Versicherungsgewerbe hat eine Abschlussrate von 3,2\%, das Investitionsgütergewerbe folgt mit 3,3\%, anschließend das Baugewerbe und die privaten Dienstleistungen ohne Erwerbscharakter mit 3,4 \% und den höchsten Wert weist mit 3,6 \% der Bereich Gartenbau, Land- und Forstwirtschaft auf.

Für rund $85 \%$ der Beschäftigten mit Neuabschlüssen traten die Tarifsteigerungen mit zeitlicher Verzögerung in Kraft. Überwiegend waren es ein oder zwei Verzögerungsmonate. Lediglich $8 \%$ der betroffenen Beschäftigten erhielten für diese Nullmonate Pauschalzahlungen, und zwar durchschnittlich $100 €$ pro Verzögerungsmonat. Die Laufzeit der neu abgeschlossenen Tarifverträge beträgt im Durchschnitt 20,7 Monate, das sind rund dreieinhalb Monate mehr als im vergangenen Jahr. Kürzere Laufzeiten weist das Baugewerbe mit 13,4 Monaten auf, längere Laufzeiten ergaben sich mit 24 Monaten im Handel und in den Bereichen Kreditinstitute/Versicherungsgewerbe sowie Gebietskörperschaften, Sozialversicherung.
Die Aussagekraft der tariflichen Abschlussrate ist begrenzt, weil sie die Laufzeit und die Lage des Tarifabschlusses im Jahresverlauf nicht in Rechnung stellt. Generell gilt: Aussagen zur Veränderung von wirtschaftlichen Kennziffern müssen auf einen festen Zeitraum normiert werden, um vergleichbar zu sein. Veränderungsraten beziehen sich in der Regel auf das Kalenderjahr. Für einen Vergleich mit den zentralen makroökonomischen Größen ist daher ausschließlich die jahresbezogene Tariferhöhung mit Bezug auf das Vorjahr methodisch zulässig und verwendbar. Nur die Erhöhung der Tarifentgelte des Berichtsjahres im Vergleich zum Vorjahr kann sinnvoll zur Veränderungsrate des Bruttoinlandsprodukts, der Lebenshaltungskosten, der Arbeitsproduktivität, der Gewinne u. ä. in Beziehung gesetzt werden, da diese ebenfalls auf Jahresbasis ermittelt werden (vgl. genauer Bispinck 2011a).

Die jahresbezogene Steigerung der Tarifverdienste bringt - anders als die Abschlussrate - die Steigerung des durchschnittlichen tariflichen Monatsentgelts des laufenden Jahres gegenüber dem Vorjahr zum Ausdruck. Einbezogen werden bei dieser Größe auch die Pauschalzahlungen, z. B. für verzögert in Kraft getretene Neuabschlüsse und zusätzliche Einmalzahlungen.

Auf der Basis der vorliegenden diesjährigen Abschlüsse (Stichtag 20.6.2013) beträgt die kalenderjährliche Erhöhung der Tarifverdienste für 2013 durchschnittlich 2,9\%. Für jene Wirtschaftszweige und Tarifbereiche, für die bereits im vergangenen Jahr oder früher Tarifanhebungen für das gesamte Jahr 2013 vereinbart wurden, errechnet sich eine jahresbezogene Steigerung von $2,6 \%$. 
Insgesamt ergibt sich für 13,2 Mio. Beschäftigte eine durchschnittliche Tarifsteigerung für das Jahr 2013 von 2,8\%. Dieses Gesamtergebnis für 2013 wird also durch die Abschlüsse aus den Vorjahren etwas nach unten gedrückt, die für 4,5 Mio. Beschäftigte gelten, während die Neuabschlüsse in diesem Jahr bislang 8,7 Mio. Beschäftigte betreffen.

Die kalenderjährliche Tarifsteigerung von voraussichtlich 2,8 \% (Tabelle 1) für dieses Jahr liegt geringfügig über der Höhe des Vorjahres (2012: 2,7 \%). Angesichts der geschätzten Preissteigerungsrate von jahresdurchschnittlich 1,6 \% wird mit dieser Tariferhöhung gesamtwirtschaftlich in diesem Jahr eine merkliche tarifliche Reallohnsteigerung erreicht. Der neutrale Verteilungsspielraum aus Preisanstieg und Produktivitätssteigerung wird voraussichtlich ausgeschöpft.

Zwischen den einzelnen Wirtschaftsbereichen ergeben sich deutliche Unterschiede (Tabelle 1). Die Spannweite variiert zwischen 2,3 \% im Bereich Kreditinstitute, Versicherungsgewerbe und 3,6 \% im Bereich Energie- und Wasserversorgung, Bergbau. Dazwischen liegen beispielsweise das Verbrauchsgütergewerbe mit 2,4\%, das Grundstoff- und Produktionsgütergewerbe mit 2,5\%, die Gebietskörperschaften, Sozialversicherung mit 2,6\%, das Investitionsgütergewerbe mit 3,0 \% und das Baugewerbe mit 3,3\%.

Der Unterschied zwischen der tariflichen Abschlussrate für 2013 von 3,2 \% und der jahresbezogenen Tarifsteigerung von 2,8 \% geht im Wesentlichen auf zwei Faktoren zurück: Zum einen treten die Tarifanhebungen häufig erst im Laufe dieses Jahres in Kraft und wirken damit nicht auf das gesamte Kalenderjahr (dies gilt gleichermaßen für die Pauschalzahlungen für Verzögerungsmonate), zum anderen gibt es mögliche Basiseffekte aus dem Vorjahr. So haben z. B. Pauschalzahlungen eine senkende Wirkung: Während sie die Summe der Tarifvergütungen im Vorjahr erhöhen, entfallen sie im Folgejahr.

Das Tarifniveau Ost/West hat sich im vergangenen halben Jahr nicht erhöht, es beträgt für die tariflichen Grundvergütungen der mittleren Gruppe auf der Basis von Tarifbereichen mit 1,6 Mio. erfassten Beschäftigten zur Jahresmitte 2013 nach wie vor $97,0 \%$.

\section{Ausgewählte Tarifrunden}

Die Tarifrunden im öffentlichen Dienst der Länder und in der Metallindustrie haben die Tarifrunde 2013 maßgeblich geprägt. Aber auch der Tarifkonflikt im Bewachungsgewerbe fand eine große Aufmerksamkeit, weil hier mit besonderer Konsequenz das Problem des tariflichen Niedriglohneinkommens angegangen wurde. Diese drei Tarifbewegungen werden im Folgenden in der zeitlichen Abfolge der Verhandlungen in Verlauf und Ergebnis dargestellt und analysiert.

\section{1 Öffentlicher Dienst - Länder}

\subsubsection{Ausgangssituation und Forderungen}

Im Kernbereich des öffentlichen Dienstes besteht seit 2005 eine Spaltung in zwei Tarifbereiche: Bund und Gemeinden auf der einen Seite und die Länder auf der anderen Seite. Die Tarifverträge laufen zeitlich seitdem nicht mehr parallel. In diesem Jahr stand der Tarifbereich der Länder auf dem tarifpolitischen Kalender, die Verträge waren Ende 2012 ausgelaufen, während die Verträge für Bund und Gemeinden noch bis Ende 2013 laufen. Der letzte Tarifabschluss für die Länder aus dem Jahr 2011 hatte neben einer Pauschalzahlung von $360 €$ für die ersten drei Monate eine zweistufige Entgelterhöhung von 1,5\% ab April 2011 und weiteren $1,9 \%$ zuzüglich eines Sockelbetrages von $17 € \mathrm{ab}$ Januar 2012 vorgesehen. Für Bund und Gemeinden gab es 2012 einen dreigliedrigen Abschluss mit Anhebungen von 3,5 \% ab März 2012 sowie zweimal je 1,4 \% ab Januar und August 2013. Damit waren zugleich auch Orientierungsmarken für die diesjährige Tarifrunde bei den Ländern gesetzt. $^{2}$

Am 11.12.2012 beschloss die ver.di-Tarifkommission ihre Forderungen:

- Entgelterhöhung um 6,5 \% mit sozialer Komponente und $100 €$ mehr für Auszubildende bei einer Laufzeit von 12 Monaten.

- Erhöhung der Feuerwehrzulage um $25 €$ und deren Dynamisierung.

- Verhandlungen über Einschränkungen befristeter Arbeitsverhältnisse.

- Verbindliche Übernahme der Ausgebildeten.

Ver.di stellte in den Mittelpunkt der Argumentation, dass die Beschäftigten des öffentlichen Dienstes (ÖD) Anschluss an die allgemeine Einkommensentwicklung halten müssten. Die Einkommensentwicklung im öffentlichen Dienst der Länder sei in den letzten Jahren deutlich hinter der privaten Wirtschaft zurückgeblieben. Auch gegenüber den Einkommen bei Bund und Kommunen bestehe ein Nachholbedarf. Nach den Berechnungen von ver.di macht er ab Januar 2013 etwa 2,25\% und ab August 2013 3,6 \% aus. Zur Finanzierung der Forderungen verwies die Gewerkschaft vor allem auf die steigenden Steuereinnahmen und die optimistischen Prognosen des Arbeitskreises Steuerschätzung.

Außerdem wurde mit der Tarifierung der Eingruppierung der Lehrkräfte zum wiederholten Mal ein Thema auf-

2 DieTarifgemeinschaft deutscher Länder (TdL) verhandelte für alle Bundesländer außer Berlin und Hessen, die 1994 bzw. 2004 aus derTdL ausgeschieden waren. Seit Januar 2013 ist Berlin zwar wieder Mitglied, führt aber gemäß einem Vertrag aus dem Jahr 2010 seine Entgelte erst bis zum Jahr 2017 an das TdL-Niveau heran. 
gegriffen, das die Gewerkschaft Erziehung und Wissenschaft (GEW) bereits seit Jahren verfolgt. Zwar gibt es seit Anfang 2012 eine neue Entgeltordnung für den TV-L, sie bezieht jedoch die Lehrkräfte nicht mit ein. Die Forderung nach einer Lehrkräfte-Entgeltordnung (L-ego) zielt darauf ab, die Eingruppierung auf Basis von Arbeitgeber-Richtlinien, wie sie seit 1961 geltende Praxis ist, durch eine tarifliche Entgeltordnung abzulösen. Mit diesem Versuch sind die ÖDGewerkschaften bereits mehrfach gescheitert.

Ein weiteres Thema der Tarifrunde waren die tariflichen Urlaubsregelungen. Die Tarifgemeinschaft deutscher Länder (TdL) hatte aufgrund einer Entscheidung des Bundesarbeitsgerichts zur Unwirksamkeit der Altersstaffelung des Urlaubs bei Bund und Gemeinden die Regelungen zum Urlaub für die Länder-Beschäftigten gekündigt. Laut ver.di wollte die TdL zum Teil bei Neueinstellungen ab 2013 und einigen Arbeitnehmerinnen und Arbeitnehmern mit arbeitsvertraglichen Änderungen nur noch einen Urlaubsanspruch von 26 Arbeitstagen statt der bislang geltenden 26 bis 30 Tage gewähren. Diese Verschlechterung lehnte ver.di ab.

\subsubsection{Verhandlungen und Ergebnis}

Die Verhandlungen begannen am 31.1.2013 mit der detaillierten Vorstellung und Begründung seitens der Gewerkschaften. Die Arbeitgeber, die erstmals mit dem Finanzminister Sachsen-Anhalts, Jens Bullerjahn (SPD), als Verhandlungsführer antraten, verwiesen vor allem auf die verfassungsrechtliche Schuldenbremse, die die Länder verpflichte, bis 2020 ausgeglichene Haushalte vorzulegen. Die Länder seien durch Personalausgaben stärker belastet als Bund oder Kommunen. Je höher der Abschluss ausfalle, umso weniger könne auf die Beamtinnen und Beamten übertragen werden. Ein Angebot legten sie in der ersten Runde nicht vor.

Die Tarifgespräche wurden am 14.2. in Potsdam fortgesetzt. Die detaillierte Diskussion des gesamten Forderungspakets führte in keinem Punkt zu einer Einigung oder auch nur einer Teileinigung. Zur Frage der Lehrkräfteeingruppierung wurde eine Arbeitsgruppe eingerichtet. Ein Angebot wurde seitens der TdL wiederum nicht vorgelegt, sodass ver.di und die anderen ÖD-Gewerkschaften zu Warnstreiks in zwei Wellen vor der dritten Tarifrunde aufriefen. Daran beteiligten sich rund 150.000 Beschäftigte. Nach Gewerkschaftsangaben lag die Zahl höher als in den Tarifbewegungen 2009 und 2011. In der dritten Verhandlungsrunde, die am 7.3. begann, konnte dann nach dreitägigen Verhandlungen am 9.3. eine Einigung mit folgenden Elementen erzielt werden.

- Erhöhung des Tarifentgelts um 2,65 \% ab 1.1.2013 sowie eine Stufenerhöhung von 2,95 \% ab 1.1.2014.

- Erhöhung der Ausbildungsvergütungen zum gleichen Zeitpunkt um $50 € / 2,95 \%$.

- Laufzeit von 24 Monaten bis 31.12.2014.

- Urlaub: von 26 bis 30 Arbeitstagen, gestaffelt nach Lebensjahren, auf 30 Tage für alle Beschäftigten ab 2013 (für Aus- zubildende 27 Tage); 1 Tag Zusatzurlaub für Beschäftigte in Zentren für Psychiatrien in Baden-Württemberg.

- Befristete Übernahme von Ausgebildeten für 12 Monate bei dienstlichem/betrieblichem Bedarf, bei entsprechender Bewährung Übernahme in ein unbefristetes Arbeitsverhältnis. - Unverzügliche Verhandlungsaufnahme nach dem 30.4.2013 über den Geltungsbereich des TV-L für die Beschäftigten an Theatern und Bühnen mit künstlerischen Tätigkeiten.

- Maßregelungsklausel.

Zu tariflichen Regelungen bei befristeter Beschäftigung waren die Arbeitgeber nicht bereit. Auch zur Tarifierung der Eingruppierung für Lehrkräfte konnte keine Einigung erreicht werden. Die GEW erklärte, dass die TdL ein Angebot vorgelegt hätte, das fast inhaltsgleich mit dem Angebot aus der Tarifrunde 2011 war und schon damals als nicht verhandelbar zurückgewiesen wurde.

Verhandlungskommission und Bundestarifkommission von ver.di bewerteten das materielle Ergebnis positiv, wenn auch das Fehlen einer sozialen Komponente bedauert wurde. „Damit halten die Länderbeschäftigten Anschluss an die Gehaltsentwicklung der übrigen Bereiche im öffentlichen Dienst und in der Gesamtwirtschaft", so bewertete der ver.di-Vorsitzende Frank Bsirske den Abschluss. Es sei ein echter Erfolg, dass für alle Länderbeschäftigten einheitlich 30 Tage Urlaub vereinbart werden konnten. In der anschließenden Mitgliederbefragung, die bis zum 9.4. durchgeführt wurde, sprachen sich bei ver.di gut $90 \%$ der betroffenen Mitglieder für die Annahme der Tarifeinigung aus.

Bei der GEW fiel die Zustimmung deutlich geringer aus, sie betrug im bundesweiten Schnitt rund $78 \%$. Nach dem Misserfolg in Sachen L-ego beriet die GEW auf einer tarifpolitischen Strategiekonferenz vom 8. bis 10.4. in Merseburg über das weitere Vorgehen. Im Ergebnis entschied sich die Gewerkschaft dafür, in Zukunft die Frage der Entgeltordnung nicht mehr mit der TdL, sondern mit den einzelnen Bundesländern zu verhandeln. Dabei soll die L-ego-Thematik dann auch mit anderen Tariffragen gemeinsam behandelt werden.

Für die Beschäftigten im öffentlichen Dienst des Landes Hessen erzielte ver.di am 15./16.4. ein Tarifergebnis, das mit einer zweistufigen Entgelterhöhung von je 2,8 \% (ab 1.7.2013 und 1.1.2014) das Volumen des bundesweiten Abschlusses erreichte. Außerdem erhalten die Beschäftigten zwei Einmalzahlungen von $450 €$ für Januar bis Juni 2013 und $225 €$ für Januar bis März 2014. Die Urlaubsregelungen entsprechen ebenfalls dem Bundesergebnis. Die Zustimmung der Mitglieder lag bei über $93 \%$.

\subsection{Metall- und Elektroindustrie}

\subsubsection{Ausgangslage und Forderungen}

Eine relativ kurze Tarifrunde mit einer intensiven Warnstreikphase und einer Abschlussrate unterhalb des Vorjahresniveaus - das sind die Charakteristika der Metalltarifverhandlungen des Jahres 2013. Wie in den vergangenen 
Jahren stand auch dieses Mal die Tarifrunde in der Metallund Elektroindustrie im Mittelpunkt des öffentlichen Interesses. Nach wie vor setzt diese Branche die Orientierungsmarke für die jeweilige Tarifrunde, auch wenn das „pattern bargaining", bei dem die anderen Branchen dem vorgegebenen Tarifabschluss der Leitbranchen mehr oder minder folgen, schon seit geraumer Zeit nicht mehr so funktioniert, wie das in früheren Zeiten der Fall war (Bispinck 2011b). Der Metallabschluss des Jahres 2012 lief Ende April 2013 aus. Er hatte eine Anhebung der Tarifentgelte um 4,3\% nach einem Nullmonat mit einer Laufzeit von 13 Monaten zum Inhalt. Außerdem einigten sich die Tarifparteien der Branche im vergangenen Jahr auf Regelungen zur Übernahme der Ausgebildeten und vereinbarten Rahmenbedingungen für den Einsatz von Leiharbeitskräften. Große Bedeutung hatte zudem der parallel abgeschlossene Tarifvertrag mit den Arbeitgeberverbänden der Zeitarbeit über Branchenzuschläge für Leiharbeitsbeschäftigte in der Metallund Elektroindustrie (Bispinck/WSI-Tarifarchiv 2013a).

In diesem Jahr zeichnete sich bereits früh eine reine Entgeltrunde $a b$, denn qualitative Themen standen nicht auf der tarifpolitischen Tagesordnung. In der gewerkschaftsinternen Forderungsdiskussion spielte neben der gesamtwirtschaftlichen Lage auch die Branchenentwicklung eine maßgebliche Rolle. Ähnlich wie bereits im Vorjahr war eine eher verhaltene wirtschaftliche Entwicklung zu beobachten. Die IG Metall betonte jedoch, dass sich die wirtschaftlichen Rahmenbedingungen für 2013 nach der Unsicherheit im zweiten Halbjahr 2012 stabilisiert hätten und sowohl für die Gesamtwirtschaft wie auch für die $\mathrm{M}+\mathrm{E}$-Industrie eine positive Tendenz aufwiesen.

Am 4.3.2013 veröffentlichte der IG Metall-Vorstand seine Forderungsempfehlung. Sie sah eine Tariferhöhung von bis zu 5,5 \% bei einer Laufzeit von zwölf Monaten vor. Dieser Empfehlung folgten die regionalen Tarifkommissionen. Die Tarifkommission des Nordverbundes (Schleswig-Holstein, Hamburg, nordwestliches Niedersachsen, Unterweser und Mecklenburg-Vorpommern) forderte explizit auch eine „soziale Komponente“. Die IG Metall bezifferte den verteilungsneutralen Spielraum für Lohnerhöhungen auf annähernd 3,5 \%, bei einer angenommenen Preissteigerungsrate von $2 \%$ und einer Produktivitätssteigerung von rund $1,5 \%$. Sie begründete ihre darüber hinausgehende Forderung mit einem „konjunkturgerechten Zuschlag“. Lohnzuwächse über den verteilungsneutralen Spielraum aus Preisund Produktivitätssteigerung hinaus schaffen, so die IG Metall, zusätzliches Einkommen. Das sei 2013 der richtige Weg. Der konjunkturgerechte Zuschlag wirke in der aktuellen Situation wie ein Stabilitätsanker für die gesamte Wirtschaft. Des Weiteren griff die IG Metall auch die Debatte um die Rolle der Löhne in Deutschland für die wirtschaftliche Entwicklung in ganz Europa auf. Sie verwies auf die große Bedeutung des Euroraums für die deutsche Exportwirtschaft und argumentierte, dass eine dynamische, auf stärkere private Nachfrage gestützte Wirtschaftsentwicklung bei uns auch den Euro-Raum stabilisiere (IG Metall 2013).
Gesamtmetall reagierte bereits auf die Forderungsempfehlung mit der erwartbaren Ablehnung. Sie bezeichnete die Empfehlung als "grotesk" und verwies darauf, dass es in der M+E-Industrie seit 2002 Lohnsteigerungen von $30 \%$ gegeben habe. Die Tarifsteigerung von 2012 sei der höchste Zuwachs seit 20 Jahren gewesen und selbst in der tiefsten Krise der Nachkriegszeit sei eine Reallohnsicherung erfolgt. Das Argument der IG Metall, über den Tarifabschluss auch die private Binnennachfrage zu steigern, sei nicht nachvollziehbar. Der Beitrag der Metallindustrie zur Stärkung der Binnenkonjunktur seien die 250.000 neuen Arbeitsplätze, die nach der Krise geschaffen worden seien. Die Metallarbeitgeber formulierten ihrerseits Anforderungen an den neuen Abschluss: Der neue Gesamtmetall-Präsident Rainer Dulger forderte eine möglichst lange Laufzeit und einen „flexiblen Abschluss, der die unterschiedliche Geschäftslage der Betriebe berücksichtigt" (Wirtschaftswoche 11.3.2013). Außerdem bezeichnete er die bestehenden Zuschlagsregelungen für Spät- und Nachtarbeit als „,nicht mehr zeitgemäß“, allerdings sei dies kein Thema der aktuellen Tarifrunde.

\subsubsection{Verhandlungen}

Insgesamt benötigten die Tarifparteien nur knapp zwei Monate und vier Verhandlungsrunden, um zu einem Ergebnis zu kommen. Die regionalen Verhandlungen starteten mit einer ersten Runde beginnend am 19.3.2013 in Bayern und der Mittelgruppe (Hessen, Rheinland-Pfalz, Saarland), gefolgt am 21. und 22.3. von Baden-Württemberg und Nordrhein-Westfalen, die in der Vergangenheit häufig als Pilotbezirke fungiert hatten.

In der zweiten Runde legten die Arbeitgeber am 19.4. in Bayern ein erstes Angebot vor, welches dann auch in allen anderen regionalen Verhandlungen der zweiten Runde präsentiert wurde, die am 25.4. in Thüringen ihren Abschluss fanden. Dieses sah nach zwei Nullmonaten (Mai und Juni) eine Erhöhung der Entgelte ab 1.7. um 2,3 \% bei einer Laufzeit von insgesamt 13 Monaten bis Ende Mai 2014 vor. Die IG Metall wies das Angebot als völlig unzureichend zurück. Berücksichtige man Nullmonate und Laufzeit, ergebe sich daraus faktisch lediglich eine Tariferhöhung um 1,9\%.

Mit Ablauf der Friedenspflicht Ende April rief die IG Metall ab dem 1.5. zu Warnstreiks auf, an denen sich bis zum 8.5. rund 400.000 Beschäftigte aus über 1.700 Betrieben beteiligten. Nachdem die dritte Verhandlungsrunde in Baden-Württemberg und Bayern am 7. und 8.5. ebenfalls ergebnislos endete, hielt die IG Metall den Druck auf die Arbeitgeber mit weiteren Warnstreiks vor der vierten Runde am 13./14.5. aufrecht. Allein in Baden-Württemberg beteiligten sich rund 70.000 Arbeitnehmerinnen und Arbeitnehmer an Aktionen und Warnstreiks. Am 13.5. trennten sich die Tarifparteien in Baden-Württemberg ohne Einigung, es gelang laut IG Metall jedoch, Lösungsmodelle zu erarbeiten. Die von den Arbeitgebern geforderte Öffnungsklausel für die betriebliche Anwendung eines Tarifergebnisses lehnte die IG Metall erneut ab. 


\subsubsection{Ergebnis}

In der vierten Verhandlungsrunde am 14.5. konnte dann für die bayerische Metall- und Elektroindustrie eine Tarifeinigung mit folgendem Inhalt erzielt werden: Nach zwei Nullmonaten (Mai und Juni) erhöhen sich die Entgelte um $3,4 \%$ ab 1.7. sowie um weitere 2,2 \% ab Mai 2014. Die gesamte Laufzeit beträgt 20 Monate bis Dezember 2014. Die Möglichkeit einer betrieblichen Abweichung von dem Ergebnis sieht der Abschluss ausdrücklich nicht vor. Der Pilotabschluss wurde zwischen dem 16. und 27.5. in allen anderen Tarifregionen übernommen.

Die Metallarbeitgeber zeigten sich mit dem Ergebnis sehr zufrieden. ${ }^{3}$ Sie stellten heraus, dass durch die lange Laufzeit Planungssicherheit für die Betriebe geschaffen worden sei. Durch die zwei Nullmonate werde die „hohe Vorbelastung“ aus dem Vorjahr „abgefedert“. Der Verhandlungsprozess habe gestrafft werden können mit einem schnellen Pilotabschluss als Ergebnis. Das erstmals nach zwei Jahrzehnten wieder ein Abschluss in Bayern erzielt wurde, trage der tarifpolitischen Bedeutung des Landes Rechnung.

Die IG Metall sah in dem Abschluss ein „gutes“ Ergebnis. „Die Beschäftigten“, so der IG Metall-Vorsitzende Berthold Huber, „werden mit 5,6 Prozent höheren Entgelten über die gesamte Laufzeit fair und angemessen an der wirtschaftlichen Entwicklung beteiligt ${ }^{\text {" }}$. Mit dieser Entgelterhöhung sichere die IG Metall den Belegschaften ein deutliches Plus. „Unsere Tarifverträge garantieren seit langen Jahren mindestens die Inflationsrate plus den gesamtwirtschaftlichen Produktivitätsfortschritt als Entgelterhöhung. Diese gute Linie schreiben wir mit dem heutigen Ergebnis fort", sagte Huber (ebd.). Die IG Metall betonte in ihrer Bewertung, dass die umfangreichen Warnstreiks maßgeblich zur Durchsetzung des Ergebnisses beigetragen hätten. Nach Angaben der Gewerkschaft beteiligten sich über 760.000 Beschäftigte aus knapp 3.000 Betrieben an Aktionen und Warnstreiks. Allerdings gab es gewerkschaftsintern auch eine kritische Debatte. Sie bezog sich auf Anlage und Koordination der Tarifrunde, auf die relativ lange Laufzeit des Abschlusses von 20 Monaten, nachdem das erste Arbeitgeberangebot zunächst nur eine Laufzeit von 13 Monaten vorgesehen hatte, und auch auf die Art der Darstellung des Ergebnisses („5,6\%“). Im Nachgang zur Tarifrunde soll in den Bezirken eine interne Diskussion über die Anlage künftiger Tarifbewegungen geführt werden.

Die in unmittelbarer zeitlicher Nähe geführten Verhandlungen bei der Volkswagen AG kamen am 28.5. zu einem vergleichbaren Ergebnis wie in der Metallindustrie. Zusätzlich konnte die IG Metall die Zahlung eines Rentenbausteins im Wert von $300 €$ durchsetzen. Dabei besteht die Möglichkeit, den Rentenbaustein in eine Einmalzahlung in Höhe von $275 €$ umzuwandeln. Damit sah sie ihr Ziel eines Ergebnisses oberhalb des Branchenabschlusses („Fläche plus“) erreicht.

\subsection{Bewachungsgewerbe}

\subsubsection{Ausgangslage}

Die Tarifrunde im Bewachungsgewerbe Nordrhein-Westfalens stellt das herausragende Ereignis des Tarifjahres 2013 dar. Forderung, Durchführung und Ergebnis überraschten die Öffentlichkeit ebenso wie die professionellen Beobachter und Tarifexperten. Das Bewachungsgewerbe (auch Wach- und Sicherheitsgewerbe bzw. Sicherheitswirtschaft) zählt zu den Niedriglohnbereichen in der deutschen Wirtschaft. Die Niedriglohnanalyse des Statistischen Bundesamtes auf Basis der Verdienststrukturerhebung 2010 weist für die privaten Wach- und Sicherheitsdienste einen Niedriglohnanteil von $60,3 \%$ aus, und selbst für tarifgebundene Betriebe liegt er danach noch bei 51,8 \%. Der entsprechende Schwellenwert betrug 10,36 € (Statistisches Bundesamt 2012).

Die Daten lassen erkennen, dass auch die tarifliche Vergütung zu wünschen übrig lässt. Dies belegt auch eine aktuelle Analyse des WSI-Tarifarchivs: $68 \%$ aller tariflichen Vergütungsgruppen der Branche lagen Ende 2012 unter einem Stundenlohn von $10 €$ und knapp die Hälfte blieb unter dem von den Gewerkschaften als Mindestlohn geforderten Stundenlohn von 8,50€. Dabei zeigt sich allerdings ein deutliches regionales Gefälle: Vor allem in den ostdeutschen Tarifgebieten aber auch in Schleswig-Holstein, Hamburg, Bremen und Rheinland-Pfalz fällt der Anteil der tariflichen Niedriglohngruppen besonders hoch aus (Bispinck/ WSI-Tarifarchiv 2013b).

Die Tariflandschaft im Bewachungsgewerbe ist vielschichtig: Tarifvertragsparteien der Branche sind ver.di und der Bundesverband der Sicherheitswirtschaft (BDSW) bzw. seine Landesverbände. Manteltarifverträge bestehen sowohl auf Landes- wie auch auf Bundesebene. Löhne und Gehälter werden dagegen nur auf Landesebene verhandelt. Dabei existieren in manchen Bundesländern eigene Verträge für bestimmte Bereiche und Beschäftigtengruppen (z. B. Geld und Wert). Die Laufzeiten der regionalen Verträge sind nicht einheitlich. In einigen Ländern wurden seit einigen Jahren keine neuen Vergütungstarifverträge mehr abgeschlossen, die alten Verträge befinden sich in der Nachwirkung bzw. es wurden Abschlüsse mit der Gewerkschaft Öffentlicher Dienst und Dienstleistungen (GÖD) des Christlichen Gewerkschaftsbundes (CGB) vereinbart. Für die Mehrheit der Tarifbereiche laufen die bestehenden Lohnund Gehaltstarifverträge bis Ende 2013. Nur in BadenWürttemberg und Nordrhein-Westfalen liefen die

3 Gesamtmetall-Informationen für die Presse 18/2013 „Planungssicherheit, Weitblick, Fairness".

4 IG Metall Pressemitteilung Nr. 28/2013 vom 15.5.2013 „Berthold Huber: Tarifergebnis garantiert Beschäftigten 5,6 Prozent mehr Geld“. 
Vergütungstarifverträge Ende Dezember 2012 aus, sodass neue Tarifverhandlungen anstanden. In Baden-Württemberg wurde bereits am 6.12.2012 ein neuer Vertrag geschlossen. ${ }^{5}$ In Hamburg enthielt der Lohntarifvertrag von 2012 eine Klausel, die Verhandlungen spätestens im November 2012 über Erhöhungen der Lohngruppe nach $₫ 5$ Luftsicherheitsgesetz (Luftsicherheitsassistenten) vorsah.

In NRW beschloss die Tarifkommission nach intensiver Diskussion im November 2012 ihre Forderungen:

$-2,50 € /$ Std. mehr für die Beschäftigten in den Lohngruppen $1-16$ und 19.

- Erhöhung der Löhne im Bereich Aviation auf $16 €$ für Beschäftigte nach $₫ \S 5,8$ und 9 Luftsicherheitsgesetz (LuftSiG).

- Erhöhung der Löhne der Werkfeuerwehr auf das Niveau des öffentlichen Dienstes.

- Erhöhung der Ausbildungsvergütungen um $100 €$.

- Erhöhung der Gehälter um $150 €$ monatlich.

- Neue Eingruppierungsregeln.

- Laufzeit von zwölf Monaten.

Umgerechnet auf den Stundenlohn in der untersten Lohngruppe von $8,15 €$ bedeuten 2,50 € eine Anhebung um 30,7 \%. Im Aviation-Bereich beträgt der Stundenlohn für Luftsicherheitsassistenten nach $\$ 5 \mathrm{LuftSiG} 12,36 €$; eine Anhebung auf $16 €$ entspricht einer Steigerung um 29,4\%. Diese Forderungen, noch dazu verknüpft mit einer Laufzeit von zwölf Monaten, waren ein überdeutliches Signal, dass die herkömmliche Begründungsstruktur für Tarifforderungen für ver.di zumindest in dieser Tarifbewegung keine Gültigkeit hatte. Es ging der Gewerkschaft nicht um einen Ausgleich für die steigenden Lebenshaltungskosten und eine Teilhabe der Beschäftigten an der steigenden wirtschaftlichen Leistungsfähigkeit (Arbeitsproduktivität). Alleiniger Maßstab war ein auskömmlicher Lohn: „Wir wollen weg von Niedriglöhnen und hin zu einem Lohn, der zum Leben reicht", hieß es in einem ver.di-Flugblatt zur Tarifbewegung.

\subsubsection{Verhandlungen und Ergebnis}

Diese Forderung traf auf einen unvorbereiteten Arbeitgeberverband. In den vergangenen Jahren hatten sich die Tarifsteigerungen in der Branche insgesamt und auch in NRW im herkömmlichen Rahmen gehalten, sie lagen unter dem gesamtwirtschaftlichen Durchschnitt. Offenkundig unter dem Eindruck des Forderungspakets legten die Arbeitgeber bereits in der ersten Verhandlungsrunde am 7.12.2012 ein Angebot vor, das folgende Punkte umfasste:

- 0,40€ mehr für die Lohngruppen 1-4, 6- 16 und 19

- 0,52€ mehr für Lohngruppe 5

$-0,75 €$ mehr für Beschäftigte nach $\$ \$ 8$ und 9 LuftSiG

$-1,14 €$ mehr für Beschäftigte nach $\$ 5$ LuftSiG

$-0,38 €$ mehr bei Löhnen der Werksfeuerwehr

- Laufzeit zwölf Monate.
$\mathrm{Zu}$ den übrigen Forderungen gab es keine Angebote. Bezogen auf die unterste Lohngruppe bedeutete das Angebot von $0,40 €$ eine Steigerung um 4,9\% und im Aviation-Bereich um 8,3 bzw. 9,2\%. Bereits dieses erste Angebot lag damit höher als jeder andere Abschluss in dieser Tarifrunde. Gleichwohl klaffte eine riesige Lücke zur gewerkschaftlichen Forderung. Ver.di forderte also eine „deutliche“ Erhöhung des Angebots. Die zweite Verhandlungsrunde am 18.12. brachte keine substanzielle Verbesserung, einen weiteren von ver.di vorgeschlagenen Verhandlungstermin lehnte der Arbeitgeberverband ab. Daraufhin sah ver.di keine realistische Verhandlungsperspektive mehr und erklärte die Verhandlungen für gescheitert.

Am 24. und 25.1. kam es zu ganztägigen Streiks an den Flughäfen Düsseldorf und Köln/Bonn sowie am 28.1. zu Arbeitskampfmaßnahmen am Kernbrennelementezwischenlager in Ahaus und bei der Werksfeuerwehr des Chemiewerks Solvay in Rheinberg. Nach Angaben von ver.di gab es eine Streikbeteiligung von bis zu $90 \%$ und mehr. In der Folge der Streiks trafen die Tarifparteien am 7.2. zu einem Sondierungsgespräch zusammen, das aber ohne Ergebnis abgebrochen wurde. Ver.di setzte daraufhin weitere Streiks an. Ganztägige Arbeitsniederlegungen fanden am 14.2. am Düsseldorfer sowie am 15. und 18.2. am Kölner Flughafen statt. Es folgte ein „Tag der Bewachung “ am 19.2. in Düsseldorf und ein weiterer sechsstündiger Streik an den Flughäfen anlässlich der Luftsicherheitstage am 21.2.

Aufgrund des wachsenden Einigungsdrucks trafen sich die Verhandlungsparteien am 26.2. zu einem Sondierungsgespräch und am 1.3. zu einer offiziellen dritten Verhandlungsrunde, die wiederum ergebnislos blieb. Den bereits zu Jahresbeginn gemachten und mehrfach wiederholten Arbeitgebervorschlag, die Schlichtung anzurufen, lehnte ver.di immer wieder mit der Begründung ab, die Positionen der Parteien lägen für ein erfolgversprechendes Schlichtungsverfahren noch zu weit auseinander. Stattdessen kam es vom 7. bis 15.3. zu mehreren ganztägigen Streiks an den beiden Flughäfen und anderen betrieblichen Kampfmaßnahmen. Am 18.3. folgte die vierte Verhandlungsrunde. Die Arbeitgeber stockten nun erstmals ihr Angebot im Bereich der Bewachung um 2 Cent auf. Für 2014 sollten die Lohnsteigerungen deutlich geringer ausfallen als im Jahr 2013. Das reichte erwartungsgemäß nicht für eine Einigung, aber die Tarifparteien verständigten sich auf eine Schlichtung unter Vorsitz des Landesschlichters von NRW, Bernhard Pollmeyer. Die Schlichtungsverhandlungen fanden am 27.3. und 5.4. statt. Der Schlichterspruch fand die Zustimmung der Arbeitgeberseite und wurde

5 Ver.di hatte in Baden-Württemberg eine Tariflohnerhöhung von $6 \%$ gefordert und für die Stundenlöhne im Bereich Aviation eine Anhebung auf 13 bzw. 13,50 €. Im Schnitt stiegen dieTariflöhne dann um 2,9\% und im Aviation-Bereich um 8,2 \% bei einer Laufzeit von zwölf Monaten. 
auch von der ver.di-Tarifkommission einstimmig angenommen. Er sah nach vier Nullmonaten (Januar bis April) die Anhebung der Lohngruppen zum 1.5.2013 sowie eine Stufenanhebung zum 1.1.2014 vor und zwar in folgender Höhe:

- Unterste Lohngruppe: + 5,8/4,4 \% (Gesamterhöhung $10,5 \%)$

- Fracht-, Personen- und Warenkontrolle an Verkehrsflughäfen: $+8,3 / 8,2 \%$ (insgesamt 17,2 \%)

- Passagierkontrolle: + 10,0/8,1 \% (insgesamt 18,9\%)

- im Durchschnitt aller Lohngruppen: + 4,7/3,9 \% (insgesamt $8,8 \%$ )

- Gehälter und Ausbildungsvergütungen: + 3,5/3,5 \%

- Erhöhung der Zulage in der Personen- und Warenkontrolle an Verkehrsflughäfen auf $1,50 €$

- Laufzeit: 24 Monate

- Verhandlungsverpflichtung für eine neue Lohnstruktur

- Wiederinkraftsetzen des Manteltarifvertrages.

Das Ergebnis führte indirekt auch zu einer Einigung im Tarifgebiet Hamburg. Dort hatte ver.di für die Luftsicherheitsassistenten ebenfalls nach mehreren Streikaktionen am 11.3. ein erstes Verhandlungsergebnis erzielt, das eine Tarifsteigerung von gut $15 \%$ beinhaltete. In einer Mitgliederbefragung sprachen sich jedoch $75 \%$ nicht für die Annahme, sondern für eine Schlichtung aus. Diese fand, nach dem Abschluss in NRW, erneut unter Vorsitz des NRW-Landesschlichters Pollmeyer statt und führte zu einem verbesserten Ergebnis von ebenfalls rund $18 \%$ Tarifsteigerung bis Ende 2014.

In NRW sprachen sich in der anschließenden Mitgliederbefragung 87,04\% für die Annahme des Schlichterspruchs aus. Ver.di bewertete den Abschluss durchweg positiv: „Durch die hohe Streikbereitschaft konnten wir eine neue Tarifpolitik in der Branche einleiten“, sagte ver.diVerhandlungsführerin Andrea Becker. Ver.di habe die Niedriglöhne in der Branche noch nicht beseitigt, konnte sie aber wesentlich stärker als in anderen Branchen anheben. Die Richtigkeit dieser Einschätzung zeigt sich insbesondere bei den für die Gewerkschaft zentralen Vergütungsgruppen: Die unterste Lohngruppe, in der laut ver.di über $70 \%$ der Beschäftigten eingruppiert sind, steigt in zwei Jahren um $10,5 \%$, die Lohngruppe der Beschäftigten in der Passagierkontrolle erhöht sich im gleichen Zeitraum sogar um 18,9\%. Diese Abschlussraten liegen zwei- bis dreifach so hoch wie die durchschnittlichen Abschlussraten der länger laufenden Tarifabschlüsse dieser Tarifrunde.

Die Gründe für diesen Erfolg sind vielschichtig: Zum einen ist es ver.di gelungen, die Forderung und vor allem ihre Begründung in der Öffentlichkeit gut zu verankern. Die Polemik der Arbeitgeber gegen die "unzumutbare“ $30 \%$-Forderung verfing nicht, weil ver.di sich auf die Prozentrechnung gar nicht erst einließ, sondern konsequent bei ihrer Grundaussage blieb, dass sie Löhne durchsetzen wolle, von denen die Beschäftigten leben können. Diese
Argumentation fiel auch insofern auf fruchtbaren Boden, weil der gesellschaftliche Unmut über sich ausbreitende Niedriglöhne und Lohndumping mittlerweile weit verbreitet ist. Das hat dazu geführt, dass die Sympathien der Öffentlichkeit und auch der unmittelbar betroffenen Passagiere lange Zeit auf Seiten der Beschäftigten blieben und es nicht zum gefürchteten Spießrutenlaufen für die Gewerkschaft kam. Zum andern nutzte ver.di erfolgreich die Durchsetzungskraft der gut organisierten und kampfstarken Beschäftigten im Sicherheitsbereich der Flughäfen für die gesamte Branche und überspielte damit die relative Schwäche im regulären Bewachungsgewerbe.

\section{Ausblick}

Ein großer Tarifkonflikt dieses Jahres ist zur Jahresmitte nicht einmal ansatzweise gelöst: Im Einzelhandel befinden sich die Tarifparteien noch mitten in der Auseinandersetzung. Ver.di hatte sich auf eine reguläre Lohn- und Gehaltsrunde vorbereitet und forderte in der Mehrzahl der Tarifbereiche Festbeträge von $1 €$ je Stunde (= 163 bzw. $165 € /$ Monat in West bzw. Ost), in den meisten anderen Bereichen $6,5 \%$ mindestens $140 €$. Die Arbeitgeber ihrerseits kündigten Ende Januar in einer bundesweit abgestimmten Aktion alle regionalen Manteltarifverträge (außer in Hamburg) und forderten eine „Modernisierung “ der Mantelbestimmungen, konkret: eine weitgehende Flexibilisierung der Arbeitszeit, eine verringerte Bezahlung der Kassiererinnen und Kassierer, die Einführung einer Niedriglohngruppe für die sogenannte Warenverräumung, den Wegfall der Möglichkeit des Durchstiegs für Ungelernte in die Gelerntengruppe und die Streichung der Zuschläge für Spät- und Nachtarbeit. Ver.di wies die Forderungen als einen massiven Angriff auf die Arbeitsbedingungen und Einkommen der Einzelhandelsbeschäftigten zurück und lehnte Verhandlungen ab. In der dritten Verhandlungsrunde am 24.6. in Baden-Württemberg legten die Arbeitgeber ein erstes Angebot über eine Tariferhöhung von insgesamt $4 \%$ über einen Zeitraum von zwei Jahren vor, knüpften es aber an die Bedingung, dass ver.di auch über Manteltarif- und Strukturthemen verhandelt.

In einigen weiteren Tarifbereichen stehen im 2. Halbjahr Lohn- und Gehaltsverhandlungen an. Ende September wird für das Maler- und Lackiererhandwerk und für das Gerüstbaugewerbe verhandelt. Ende Oktober laufen die Verträge für den Garten-, Landschafts- und Sportplatzbau sowie für die Leih-/Zeitarbeit aus. Für die Leih-/Zeitarbeit laufen die Verhandlungen bereits. Ende Dezember 2013 laufen dann u. a. die Verträge für die Druckindustrie, die private Abfallwirtschaft und weitere kleinere Tarifbereiche aus. Eine grundsätzliche Veränderung des bislang beobachtbaren Trends der Tarifentwicklung steht nicht zu erwarten.

Die Tarifrunde 2014 wird zu Beginn bestimmt werden von den Verhandlungen in der chemischen Industrie, 
hier laufen die regionalen Verträge zwischen Ende Dezember 2013 und Ende Februar 2014 aus, und im öffentlichen Dienst (Bund und Gemeinden). Größere Branchen folgen dann mit dem Bankgewerbe, dem Bauhauptgewerbe (Ende April) und der Eisen- und Stahlindustrie (Ende Mai). Die Verträge in der Metallindustrie laufen bis zum Jahresende 2014.

\section{LITERATUR}

Bispinck, R. (2011a): Welche materiellen Wirkungen hat ein Tarifabschluss?, Erläuterungen zurTarifstatistik, in: Elemente qualitativer Tarifpolitik (71), Düsseldorf

Bispinck, R. (2011b): Pay in the 2000s: development and outcomes, WSI Report (6), Düsseldorf

Bispinck, R./WSI-Tarifarchiv (2013a):Tarifpolitischer Jahresbericht 2012. HöhereTarifabschlüsse und Erfolge bei Leiharbeit und Ausbildung, in: WSI-Mitteilungen 66 (2), S. 117-125, http://www.boeckler.de/wsimit_2013_02_bispinck. pdf

Bispinck, R./WSI-Tarifarchiv (2013b): Tarifliche Vergütungsgruppen im Niedriglohnbereich 2012. Eine Untersuchung in 41 Wirtschaftszweigen, Elemente qualitativer Tarifpolitik (75), Düsseldorf

Brenke, K./Wagner, G. (2013): Ungleiche Verteilung der Einkommen bremst das Wirtschaftswachstum, in: Wirtschaftsdienst 93 (2), S. 110-116

Bundesagentur für Arbeit (BA) (2012): Der Arbeits- und Ausbildungsmarkt in Deutschland, Monatsbericht Dezember und Jahr 2012, Dezembe
IG Metall (2013): Argumente zurTarifrunde, Frankfurt a. M., online, http://www igmetall.de/cps/rde/xchg/internet/style.xsl/argumente-zur-tarifrunde-2013-11428.htm

Statistisches Bundesamt (2012): Niedriglohn und Beschäftigung 2010, Begleitmaterial zur Pressekonferenz am 10. September 2012 in Berlin, Wiesbaden Stein, U./Stephan, S./Zwiener, R. (2012): Zu schwache deutsche Arbeitskostenentwicklung belastet Europäische Währungsunion und soziale Sicherung, IMK Report (77), November

\section{AUTOREN}

REINHARD BISPINCK, Dr., ist Wissenschaftler im Wirtschafts- und Sozialwissenschaftlichen Institut (WSI) und Leiter des WSI-Tarifarchivs in der HansBöckler-Stiftung.

@ reinhard-bispinck@boeckler.de

GÖTZ BAUER, MERLE FÖHR, ULRICH SCHMIDT, MONIKA SCHWACKE-PILGER, KATHRIN SONNEN, ANDREA TAUBE und MONIKA WIEBEL sind Sachbearbeiterinnen und Sachbearbeiter im WSI-Tarifarchiv. 